\title{
Cultivating Mindfulness in Schools to Promote Well-Being
}

Tyler L. Renshaw ${ }^{1}$, Sean N. Weeks ${ }^{1}$, Anthony J. Roberson ${ }^{2}$, Shelley R. Upton ${ }^{1}$, John D. Barr ${ }^{1}$, Mary L. Phan ${ }^{1}$, \& Caleb D. Farley ${ }^{1}$

${ }^{1}$ Department of Psychology, Utah State University

${ }^{2}$ Cypress-Fairbanks Independent School District, Texas

\section{Author Note}

This is a pre-publication version of a chapter intended for inclusion in the forthcoming Handbook of Positive Psychology in Schools (3rd ed.), edited by K. Allen, M. J. Furlong, D. VellaBrodrick, and S. M. Suldo. This version is not the copy of record and may not exactly replicate the authoritative, published version of the chapter.

The authors declare no conflicts of interest related to this work.

Correspondence should be sent to Tyler Renshaw at tyler.renshaw@usu.edu. 


\begin{abstract}
This chapter orients readers to the landscape of mindfulness and mindfulness-based intervention (MBI) in schools. First, definitions and examples of the key constructs are given: mindfulness and $\mathrm{MBI}$. Next, a discussion is provided on the empirical support and contemporary issues surrounding two aspects of evidence-based practice related to mindfulness in schools: $\mathrm{MBI}$ and mindfulness measurement. Following, major considerations are highlighted related to the practical application of mindfulness in schools, focusing on the benefit of a multireed service delivery and the relevance of diversity and developmental considerations when applying mindfulness for the benefit of all in schools. On the whole, this chapter offers a realistic yet optimistic perspective on mindfulness and $\mathrm{MBI}$-suggesting a promising, empirically supported approach for promoting desirable positive psychological functioning among students and their caregivers. Yet much is still unknown; thus, much work remains to inform a thoroughgoing evidence-based approach to using mindfulness and $\mathrm{MBI}$ in schools.
\end{abstract}

Keywords: mindfulness, mindfulness-based intervention, wellbeing, school mental health 


\section{Cultivating Mindfulness in Schools to Promote Well-Being}

Mindfulness is a contemporary psychological construct that has origins in Buddhism's ancient traditions and other Eastern worldviews. It was not until the late 1970s that mindfulness transitioned from Eastern philosophy to Western science. This secularizing shift was spurred by the work of Jon Kabat-Zinn (1990), who claimed that mindfulness "can be learned or practiced ... without appealing to [Eastern] culture or Buddhist authority to enrich or authenticate it" (p. 12). Kabat-Zinn and colleagues initiated the mindfulness-as-intervention movement by testing the effects of mindfulness-based practices for reducing stress with medical patients experiencing chronic pain. The success of these early studies led, over time, to the eventual generalization of mindfulness-based intervention (MBI) as an evidence-based practice for reducing suffering and promoting well-being with diverse individuals across a variety of service settings. The chapter focuses specifically on the application of mindfulness and $\mathrm{MBI}$ within school settings to promote desirable psychological functioning among students and their caregivers. This chapter's previous version focused on cultivating mindfulness with students (Renshaw \& O'Malley, 2014). The present work expands the reach of MBI to teachers and parents, as schools are becoming increasingly common hubs for intervening with caregivers - both for their own sake (i.e., direct benefit) and for the sake of the youth they care for (i.e., indirect benefit).

To set the stage for mindfulness in schools, definitions and key concepts are discussed. Following, we highlight the landscape of evidence-based practice related to $\mathrm{MBI}$ and measuring mindfulness, pointing out key considerations and directions for future research. Practical applications of mindfulness and $\mathrm{MBI}$ in schools are then discussed, emphasizing its potential 
within a multitiered system of supports (MTSS) for promoting mental health and socialemotional well-being as well as its promise as an indirect service delivery approach with caregivers. Finally, essential diversity and developmental considerations are identified when using mindfulness in schools to address health disparities and optimize the potential benefit of $\mathrm{MBI}$ for all students.

\section{Definitions and Key Concepts}

The most common definition of mindfulness is likely that provided by Kabat-Zinn (1994), who described it as "paying attention in a particular way: on purpose, in the present moment, and nonjudgmentally" (p. 4). Smalley and Winston (2010) offered a similar definition of mindfulness, describing it as a state of consciousness wherein one "observ[es] physical, emotional, and mental experiences with deliberate, open, and curious attention" (p. 11). Brown et al. (2007) have defined mindfulness along the same lines as "receptive attention to and awareness of present events and experience" (p. 212). To consolidate the essence of these similar definitions, a few different distillations of the core components of mindfulness have been offered in other works (e.g., Renshaw, 2012; Renshaw \& Cook, 2017; Renshaw et al., 2017). Yet, this chapter prefers the consensus definition from a renowned group of mindfulness scholars (Bishop et al., 2004), who describe the construct as "the self-regulation of attention so that it is maintained on immediate experience," which is accompanied by "a particular orientation towards one experience in the present moment" (p. 232). Throughout this chapter, we refer to these two core components of mindfulness as present moment awareness (PMA) and responding with acceptance (RWA). Instead of thinking of PMA and RWA as states of mind or qualities of consciousness, we suggest that it is more useful to think of them as actions, 
behaviors, or skills, which can be learned and therefore trained in schools like any other socialemotional skill (Renshaw, 2020).

The first core component of mindfulness, PMA, is the action or skill of orienting one's attention to experiences in the here and now. Noticing what one is thinking in the momentwhether helpful or unhelpful-and tuning in to one's feelings-whether desirable or undesirable-are common, everyday applications of PMA. Non-applications or opposites of PMA include the just as common, everyday experiences of ruminating about one's past mistakes or forecasting about one's future (but not yet actual) missteps. Examples of proper PMA include an adolescent noticing the "heartbreak" they experience following a romantic break-up, the "butterflies in their stomach" before taking a math test, and the encouraging selftalk running through their mind while succeeding at a well-rehearsed task (e.g., "Wow! I'm really good at this!"). Non-examples of PMA, then, include the same adolescent retracing the events leading up to the romantic break-up, planning ways to effectively avoid the math test to relieve the "butterflies in their stomach," and becoming completed absorbed in-or lost in flow with (see Chapter 19 of this volume) - the familiar task at hand. These non-examples of PMA are not necessarily unhelpful actions, as these same experiences can sometimes be useful for problem-solving or even enjoyable for their own sake. The point is simply that these examples are not mindfulness, as PMA requires being directly in touch with one's experiences in the here and now, as opposed to planning around or being absorbed in these experiences.

The second core component of mindfulness, RWA, is the behavior or skill of engaging in one's experiences in a welcoming and open way. As Renshaw (2020) puts it, "the core features of RWA are being curious about the nature of one's experiences in the present moment while 
either (a) intentionally staying in contact with whatever one is experiencing for however long it happens to last or (b) persisting in accomplishing a valued activity with which the experience may potentially interfere" (p. 146). The opposite of RWA is an avoidant response, which functions to change the content of one's experiences or reduce the amount of time one is in contact with such experiences. To continue the scenarios from earlier, examples of proper RWA include an adolescent staying in touch with the "butterflies in their stomach" while persisting and engaging with the math test, or mundanely noticing the onset and offset of their "heartbreak" throughout the school day. Non-examples of RWA, then, might include this same adolescent complaining to the teacher that they are "sick" and requesting to visit the nurse's office (to escape the math test), or picking up one's phone to check notifications or cruise social media for a quick distraction whenever feelings of "heartbreak" arise. Again, these nonexamples or RWA are not inherently or always harmful. Rather, the point is that they are not mindfulness, as they involve actions that are antithetical to staying in touch with one's experiences in a welcoming and open way.

With these two core components of mindfulness-PMA and RWA-in hand, we can now move to define the notion of mindfulness-as-intervention or $\mathrm{MBI}$. In short, $\mathrm{MBI}$ refers to any approach or procedure that aims to train PMA and RWA to reduce suffering or improve wellbeing. As Renshaw (2020) puts it, the critical feature of MBI "is not necessarily the structure of the intervention [per se] but rather the functions of the intervention" (p. 147). Thus, any intervention that improves PMA and RWA might be considered an MBI. Various techniques and treatment packages have been developed toward this end. $\mathrm{MBI}$ is currently a broad umbrella category of interventions, accounting for a variety of approaches ranging from relatively 
standardized and narrow treatment approaches (e.g., mindfulness-based stress reduction; Kabat-Zinn, 1994) to more flexible and integrative treatment approaches (e.g., acceptance and commitment therapy; Hayes et al., 1999). MBI also include prevention-oriented socialemotional learning curricula for educational use (e.g., Learning to Breathe; Broderick, 2013), and even brief, focused procedures targeting specific problems and populations (e.g., Soles of the Feet; Felver \& Singh, 2020). Despite their differences, MBIs tend to share common elements, such as formal mindfulness meditations (e.g., mindful breathing, mindful body scan) and applied or everyday mindfulness exercises (e.g., mindful eating, mindful movement). Other common elements of MBIs include metaphorical exercises to help with intuiting mindfulness (e.g., leaves on the stream, taking your mind for a walk) and formal psychoeducation about how mindfulness works to affect emotional regulation and positive behavior change. What is becoming increasingly apparent throughout the last decade is that-despite their composition or complexity-MBIs demonstrate empirical support for uses that are relevant to schools. The next section overviews what is known, so far, about the effectiveness of MBI with youth and caregivers, with a specific focus on students, teachers, and parents.

\section{Empirical Support for Mindfulness-Based Interventions}

\section{MBI with Children and Adolescents}

Over the past 20 years, the literature on MBI with children and adolescents has proliferated (Black, 2015). While many scholars suggest that the research base is still relatively young, several recent meta-analyses synthesizing effect sizes from primary studies provide a reliable estimate of the overall effectiveness of $\mathrm{MBI}$ with students and youth. This section of the chapter reviews evidence gathered from eight meta-analytic reviews, which have been 
discussed by Klingbeil et al. (in press) and others (Borquist-Colon et al., 2019; Carlsey et al., 2017; Dunning et al., 2019; Kallapiran et al., 2015; Klingbeil et al., 2017a, 2017b; Zenner et al., 2014; Zoogman et al., 2015). Taken together, we believe the outcomes of these eight metaanalytic reviews suggest that the use of MBIs in schools and with youth is generally safe and effective for improving various desirable psychological outcomes. Nevertheless, much work remains to optimize $\mathrm{MBI}$ for efficient, acceptable, and sustainable use in schools.

According to these several available meta-analyses, the majority of MBIs currently used in schools are modeled after the following approaches: mindfulness-based stress reduction (MBSR; Kabat-Zinn, 1994), mindfulness-based cognitive therapy (MBCT; Segal et al., 2002), acceptance and commitment therapy (ACT; Hayes et al., 1999), dialectical behavior therapy (DBT; Linehan et al., 1991), and mindfulness-based yoga programs (MY; e.g., Dariotis et al., 2017). Most school-based MBI used formalized interventions through third-party providers or research staff, although some included programs explicitly designed for implementation by teachers or other staff at the school. Interestingly, Klingbeil et al. (2017) found no difference in average effect sizes from studies conducted in schools versus those carried out in community or clinical settings-suggesting that school-based $\mathrm{MBI}$ is just as effective as $\mathrm{MBI}$ with youths in other settings.

Perhaps the most striking conclusion drawn from the several available meta-analyses of $\mathrm{MBI}$ with children and adolescents is how broad the range of outcomes is that have been positively affected by mindfulness training (Borquist-Colon et al., 2019; Carlsey et al., 2017; Dunning et al., 2019; Kallapiran et al., 2015; Klingbeil et al., 2017a, 2017b; Zenner et al., 2014; Zoogman et al., 2015). To start with, when measuring the mechanism of mindfulness itself, MBI 
effect sizes have ranged from small to medium, are positive, and have moderated the effects of mental health outcomes. In terms of reducing stress, negative emotions, and internalizing problems, $\mathrm{MBI}$ effect sizes were positive and ranged from small to moderate. Furthermore, $\mathrm{MBI}$ has been shown to have small to moderate effect sizes for increasing subjective well-being and related positive psychological variables, including positive emotions, coping strategies in response to stress, self-esteem, and self-concept. Although research is less robust in terms of $\mathrm{MBI}$ effects on undesirable behavioral outcomes-with far fewer studies targeting this domain-available evidence indicates small to moderate effect sizes for reducing externalizing and disruptive behaviors, with small effects on reducing aggression and hostility. Maybe the most inconsistent findings, so far, are in the area of $\mathrm{MBI}$ effects on prosocial behavior and social skills, with some reviews yielding nonsignificant outcomes while others showed small positive effects. Furthermore, multiple meta-analyses indicate that school functioning and academic achievement are improved by $\mathrm{MBI}$, with small positive effects. Ultimately, it seems safe to say that $\mathrm{MBI}$ is useful in schools for improving a variety of psychological and educational outcomes for children and adolescents. However, given the variability in effect sizes observed across meta-analyses, it also seems wise to conclude that these effects are likely small, especially for classroom-based MBI implemented as universal supports (Renshaw, 2020).

Beyond indicating generally positive effects, the available literature on $\mathrm{MBI}$ with children and adolescents also suggests several limitations. For example, barriers to $\mathrm{MBI}$ provision within schools have been identified, including scheduling conflicts, lack of resources or available spaces, coordinating student schedules, and securing buy-in from parents and teachers (Dariotis et al., 2017). Interestingly, youths' age has not significantly moderated the 
overall effect sizes of $\mathrm{MBI}$ in meta-analyses. However, some studies have found differences by age, suggesting the need to explore further demographic moderators. For example, MBIs produced stronger effects on executive functioning with older youths and reduced externalizing behaviors with larger effect sizes with younger youths. Moreover, effect sizes for mental health outcomes were generally larger for participants in late adolescence, with effects reducing to non-significant levels with younger children. Furthermore, some primary studies within the meta-analyses found that MBIs may be more effective for females and that they worked equally well across youth with varying clinical diagnoses, and with and without educational disabilities. We, therefore, echo the calls made elsewhere, recommending that further rigorous research is warranted in order to understand potential moderators that might inform precise use of MBI in schools (Renshaw, 2020; Renshaw \& Cook, 2017; Renshaw et al., 2017).

\section{MBI with Teachers and Parents}

As the research base for youth-focused MBI has grown in recent years, so too has the evidence for using $\mathrm{MBI}$ with teachers and parents. Much of the investigations with this population have honed-in on the effectiveness of mindfulness training for positively altering mental health outcomes centered around psychological well-being and distress. Thus far, the evidence suggests that MBI offers some improved outcomes for both parents and teachers. Yet, this conclusion should be held more tentatively than the upshot of MBI with children and adolescents, which we believe indicates broader and more consistently positive effects compared to $\mathrm{MBI}$ with caregivers. It is noteworthy that much of the theory underlying providing $\mathrm{MBI}$ with caregivers focuses on their potential indirect benefits to youth. However, evaluation studies have focused almost entirely on the direct benefit of $\mathrm{MBI}$ for caregivers themselves- 
recognizing that caregiving can have substantial psychological costs.

Teachers are crucial contributors to society, and their occupation is often demanding and stressful (Kyriacou, 2001). Teachers experience high burnout and job transition (Markow \& Pieters, 2012), with recent data suggesting that $50 \%$ of new teachers will not be teaching within five years (Markow \& Cooper, 2008). Clearly, then, stress management skills and psychological support are germane considerations for these caregivers. MBI has been developed as one potential means toward this end. A recent synthesis of relevant research with teachers suggest that MBIs produce improvements in stress levels and psychological health, including higher resiliency levels, which may help address teacher burnout (Thayer et al., in press). A recent meta-analysis on this topic by Klingbeil and Renshaw (2018) indicated that MBI produced moderate improvements in teacher psychological well-being, mindfulness skills, and classroom practices. Overall, this emerging evidence suggests that $\mathrm{MBI}$ may help enhance teachers' stress management skills and improve their psychological health. Yet, the evidence base does not provide any information about how MBI might indirectly benefit students in the classroom.

In addition to serving as a setting for intervening with educators, schools are also common hubs for targeting youths' primary caregivers: parents and guardians. Tasked with the vital responsibility of raising youths, parents and caregivers often experience emotional adversity and may benefit from direct training in coping skills to overcome these challenges. It is well-understood that the parent-child relationship within the home environment is one of the most critical factors for healthy child development and well-being. Specifically, insufficient or neglectful parental care is a common predictor of youths' future problems (Felitti et al., 1998). In response to these realities, a large number of parent training programs have been created. 
However, research suggests that these programs are less effective for caregivers who struggle with mental health issues or significant stress, primarily because they are less likely to complete the program and implement these practices at home (van den Hoofdakker et al., 2010). MBI with parents has been offered as a potential solution to address this concern.

Recent syntheses of the research suggests that MBI with parents has produced significant decreases in parental stress, depression, anxiety, and significant increases in selfreported mindfulness skills, with effect sizes ranging from small to medium (Frantz et al., 2018; Taylor et al., 2016). Other nonexperimental studies investigating the value of mindful parenting have demonstrated that caregivers reporting higher levels of mindfulness employ more helpful and less problematic parenting practices than those with lower levels of mindfulness (Williams \& Wahler, 2010; de Bruin et al., 2014). Congruent with the evidence base with teachers, the available research supporting the use of $\mathrm{MBI}$ with parents suggests that this approach to intervention may be useful for addressing parental psychological health and cultivating nurturing familial environments. Yet, it has revealed little about how MBI with this population might indirectly improve youth outcomes. We, therefore, reiterate the calls of others to both strengthen and expand research in this area, with the goal of understanding both $\mathrm{MBI}^{\prime} \mathrm{s}$ potential direct and indirect impacts with caregivers (Klingbeil \& Renshaw, 2018).

\section{Empirical Support for Measuring Mindfulness}

In addition to evidence-based intervention, another-often overlooked-consideration when applying mindfulness in schools is the available empirical support for mindfulness measures. Using appropriate assessment tools is a critical component in effectively implementing and evaluating interventions, and MBIs are no exception. The use of strong 
measures allows for validating the purported mechanisms of change driving $\mathrm{MBI}$ and reveals if changes in mindfulness proceed observed changes in outcomes of interest, strengthening the evidence for causation (see Nock, 2007). The development and study of mindfulness assessment tools intended for youths have recently expanded considerably. Although these measures vary in a variety of important theoretical ways, most share a common self-report rating format with respondents indicating the relative-frequency of-or degrees of agreement with-statements focusing on measuring trait (or dispositional) mindfulness.

Given the mindfulness core components model presented earlier in this chapter, youths' trait mindfulness might be best understood as the regularity with which one experiences PMA and RWA during everyday life. In other words, not only may people be considered more or less mindful at a particular moment in time (i.e., state mindfulness), but they might also be described as more or less mindful individuals most of the time (i.e., trait mindfulness). Similar to other survey instruments measuring state-trait distinctions, mindfulness measures were originally developed with university students and adult-aged samples. In recent years, however, trait mindfulness surveys have been successfully tested with youth samples and shown to covary with valued outcomes (Goodman et al., 2017). However, with few exceptions, such as the State Mindfulness Scale for Physical Activity (Ullrich-French et al., 2017), trait mindfulness surveys outnumber state measures (Goodman et al., 2017). We suggest this discrepancy in measurement approaches is far from trivial, as the primary focus of $\mathrm{MBI}$ is developing PMA and RWA right now, which relates more closely to state views of mindfulness than trait views. Yet, because trait mindfulness measures are the primary tools available, mindfulness research with youth and in schools has relied almost exclusively on trait measurements (Goodman et al., 
2017). The present understanding of the evidence supporting the processes underlying $\mathrm{MBI}$ is thus based on a construct indirectly related to its purported mechanism of change (Thompson \& Waltz, 2007).

The concerns with the current measurement paradigm are less about the strength of traditional reliability and validity evidence of particular scales and more about the lack of theoretical coherence between what these instruments measure and how they are used. To understand the true strength of $\mathrm{MBI}$ evidence with youth, it is important to recognize how mindfulness is typically measured and how conclusions from mindfulness studies depend, in part, on the particular instrument chosen to assess the construct. Although an in-depth analysis of available instruments is beyond this chapter's scope, we highlight features of several trait mindfulness measures to provide interested readers a starting point to seek information about their theoretical and psychometric strengths and weaknesses (see Eklund et al., 2017; Grossman, 2011; Goodman et al., 2017). The previous edition of this chapter (Renshaw \& O'Malley, 2014) identified and discussed two primary trait mindfulness surveys: the adolescent version of the Mindful Attention Awareness Scale (MAAS-A) and the Child and Adolescent Mindfulness Measure (CAMM). The MAAS-A and CAMM remain the most frequently studied instruments and are commonly used as convergent validity measures of youth mindfulness in the research of other mindfulness tools (e.g., Johnson et al., 2017; Levinson et al., 2014; West, 2008). These two measures are reviewed below first, followed by an expanded review of several other trait mindfulness scales developed in recent years.

\section{Trait Mindfulness Assessment Tools}

The CAMM is a 10 -item, self-report mindfulness measure for youth ages $10-17$ years 
(Greco et al., 2011) based on a multidimensional conceptualization of mindfulness-acting with awareness, accepting without judgement, and observing-used by the adult Kentucky Inventory of Mindfulness Scale (KIMS). All items are reverse coded to produce a global mindfulness score. Two other commonly used youth mindfulness measures are adapted from the MAAS (Brown \& Ryan, 2003) and reflect a single facet of mindfulness: being present. These are the MAAS-A (for adolescents) and the MAAS-C (for children). The MAAS-A is a 14-item measure for ages 14-18 years (Brown et al., 2011), whereas the MAAS-C is a 15-item measure for ages 9-13 years (Lawlor et al., 2014). Item content is nearly identical, with the MAAS-A excluding an item related to driving (i.e., "I drive places on 'automatic pilot'...") and the MAAS-C changing the language of that same item to "I walk into a room, and then wonder why I went there." Like the CAMM, all MAAS items are reverse-coded and summed to produce a single total score representing mindfulness.

Newer and less commonly studied self-report measures feature multidimensional factor structures and produce multiple scores. These include the Mindful Thinking and Action Scale for Adolescents (MTASA), the Comprehensive Inventory of Mindfulness ExperiencesAdolescents (CHIME-A), the Adolescent and Adult Mindfulness Scale (AAMS), and the Mindfulness Inventory for Children and Adolescents (MICA). The MTASA (West et al., 2005) is a 32-item measure appropriate for ages 11-19 years, which uses a mix of positively and negatively worded items. The negative items are reverse-coded to produce an overall mindfulness total score and four subscale scores. The CHIME-A is a 25-item measure for adolescents that uses both positively and negatively worded items derived from the adult version of the CHIME (Johnson et al., 2017). Total scores are calculated for each of the eight 
specific domains, but the authors recommend against calculating a global total mindfulness score.

The AAMS, on the other hand, is a 19-item measure that has been investigated with individuals from ages 9-25 years and recommended for use with individuals 11 years and older (Droutman, 2018). Item content covers four domains and has mixed valence. The authors recommend averaging items within each subdomain to obtain subscale scores, followed by summing the averages to obtain a higher-order overall mindfulness score. Furthermore, the MICA is a 25-item measure appropriate for youth ages 8-18 (Briere, 2011). Items are both positively and negatively worded and cover five subdomains. Importantly, in contrast with the other measures described above, the MICA's structural characteristics and construct validity have not been formally evaluated in an empirical study. Finally, to our knowledge, only one measure - the Mindful Student Questionnaire (MSQ) - uses all positive wording, tapping the presence of mindful experience rather than the lack thereof (cf. CAMM and MAAS). The MSQ is also the only instrument developed explicitly for use within educational contexts to measure school-specific mindfulness experiences, yielding both PMA and RWA scores that have been validated for use with students in grades 6-8 (Renshaw, 2017).

\section{Future Directions and Alterative Assessment Approaches}

As stated earlier, the measures described above share a common self-report format. However, they differ significantly in underlying theory, psychometric qualities of scores, and contexts in which their use is justified. It is critical, then, that these instruments are not considered interchangeable but rather as unique tools producing related yet distinct scores. These differences across measures create challenges for evaluating MBI evidence, as the 
meaning of change in process or mechanism scores will depend on what tools were used. Instead of relying on unidimensional mindfulness measures, we recommend multidimensional measurements to identify the mechanisms for changing or mediating responses to $\mathrm{MBI}$. We also recommend that future research should focus on investigating the interrelationships among these trait measures and the value-added effects of these measures for predicting valued outcomes. Such findings may inform an evidence-based approach for matching the most useful mindfulness assessment tools with desired MBI outcomes. Additionally, given the development of competing mindfulness frameworks vying for attention in schools, such as psychological flexibility (Greco et al., 2008) and self-compassion (Neff et al., 2021), more empirical attention should be paid to exploring the differences (or lack thereof) among these seemingly synonymous constructs.

Although self-report rating scales administered before and after an intervention (summatively or as progress-monitors) is the dominant modality for assessing youth mindfulness at present, some other measurement approaches from the adult literature have also been proposed for youth. One suggested method involves experiential reports sampled throughout a given period (Moore et al., 2016), such as a school day. This approach maintains self-report ratings but allows for data to be collected outside the immediate context of an $\mathrm{MBI}$ to give more detail about the stability of mindful experience over time. Moreover, the wording of items within an experience-sampling measurement approach might be tailored to tap trait or state mindfulness constructs, increasing potential application for targeted assessment.

Some other proposed methods for assessing mindfulness break from self-reports entirely. One promising non-survey method is the objective breath counting tasks (BCT), which 
may have utility as both an assessment of PMA and a mindfulness skill-building exercise (Levinson et al., 2014). Research shows BCT scores have small to moderate correlations with mindfulness survey scores, suggesting potential incremental validity beyond traditional rating scales. BCT scores have also shown some discriminate validity with similar constructs as well as predictive validity with more objective measures, such as discerning between individuals that have and have not received MBI (Levinson et al., 2014; Wong et al., 2018). Notably, the direct construct representation of BCT is narrowly focused on PMA and only indirectly associates with the other core mindfulness component, RWA. Translational research is still needed to investigate to what degree and for what applications non-survey methods, like the BCT, are defensible for use with youth and in schools. Yet these approaches offer the possibility of expanding the variety of mindfulness assessment tools available for informing and evaluating school-based MBI. Given that strong validation evidence supporting these alternative methods is likely a ways off, we strongly recommend that current applications of non-survey methods in schools be considered adjuncts to-not replacements for-traditional (albeit limited) trait mindfulness measures.

\section{Key Consideration for Using Mindfulness in Schools}

So far in this chapter, mindfulness and $\mathrm{MBI}$ have been defined and readers have been oriented to the landscape of evidence-based practice related to $\mathrm{MBI}$ and mindfulness measures. Moreover, mindfulness has be conceptualized as a positive psychological disposition or skillset that is amenable to empirical measurement and effective intervention, similar to other constructs covered in this volume (e.g., optimism, Chapter 9; empathy, Chapter 13; growth orientations, Chapter 21). Although the research to date is promising, much is still 
unknown; thus, much work remains to be done. Readers interested in discussion of future directions for mindfulness and $\mathrm{MBI}$ in schools are encouraged to consult other works on this topic, which offer directions toward this end (e.g., Klingbeil \& Renshaw, 2018; Klinbgeil et al., 2017; Renshaw, 2020; Renshaw \& Cook, 2017; Renshaw et al., 2017). To round out this chapter, we highlight key considerations for using mindfulness and $\mathrm{MBI}$ in schools. Although much could be considered, two areas of practice are emphasized that we believe are especially beneficial for progressing mindfulness applications in schools: integrating MBI within multitiered systems of support (MTSS), and diversity and developmental considerations for informing equitable and acceptable use of $\mathrm{MBI}$ in schools.

\section{Integrating MBI within MTSS}

Schools using social-emotional learning (SEL) or positive behavior interventions and supports (PBIS) are likely familiar with MTSS as an organizing framework for prevention and intervention. The standard MTSS model consists of three tiers-universal, targeted, and intensive levels - that function to calibrate preventative and proactive supports with student needs based on the intensity of the presenting problem, responsiveness to intervention, and resource availability. Other works have suggested that an MTSS framework could provide a viable means for implementing and distributing $\mathrm{MBI}$ in schools (e.g., Felver et al., 2013; Renshaw et al., 2017). A few guidelines for educators and school-based practitioners are provided below toward this end.

Foremostly, most mindfulness-based programs are designed for universal (or classwide) implementation, focusing on training basic mindfulness skills to promote well-being and improve all students' academic success. There are currently several available schoolwide 
mindfulness programs offering semi-standardized classroom curricula (e.g., MindUp;

https://mindup.org) and consultancy services to train educators and support implementation (e.g., Mindful Schools; https://www.mindfulschools.org). One example of an evidence-based MBI commonly used at this tier is Learning to BREATHE (L2B; Broderick, 2013), which uses the BREATHE acronym (i.e., body, reflections, emotions, attention, tenderness, and health habits) as an implementation framework. Given that the bulk of evidence for $\mathrm{MBI}$ exists at the universal level (Klingbeil et al., 2017), educators and other school-based practitioners integrating $\mathrm{MBI}$ within MTSS can do so with relative confidence at this tier, as long as they select a program that has demonstrated empirical support. Readers are referred back to the several meta-analyses mentioned above, which provide detailed information about the available evidence for specific approaches and curricula for universal MBI. To prevent redundancy among school programming approaches, universal MBI can be conceptualized as an SEL approach, with PMA and RWA considered core social-emotional competencies (Lawlor, 2016).

When it comes to integrating MBI within the other tiers of MTSS, there is, unfortunately, substantially less evidence available to guide educators and school-based practitioners. At the targeted level, mental health or SEL-type interventions are usually provided in small group settings that address a common dilemma or shared problems among students with identified risk. Although mindfulness curricula, such as L2B, may be effectively implemented at the targeted level with a subset of students, there are fewer evidence-based options at this tier. Theoretically, this tier within MTSS provides a good avenue for more specific and intensive mindfulness training and psychoeducation. Practitioners may consider a few emerging options 
in this area, including (a) the DNA-V model of ACT for SEL (Rayner et al., 2017), (b) more intensive applications of MBSR (e.g., Biegel et al., 2009), and (c) the group-based version of MBCT with youth (Semple et al., 2010). At the intensive level within MTSS, students typically receive individualized supports and specific interventions to target severe problems that cannot be precisely addressed within group or classroom settings. Although individualized MBI having been thoroughly researched in clinical settings with adults, the evidence base still lacks for guiding applications with youths in schools. Generalizing from the best available evidence in clinical settings, however, we suggest practitioners consider using MBSR, ACT, MBCT, or DBT as $\mathrm{MBI}$ at the intensive level. Sufficient practical guidance for applying these approaches with youths has been provided elsewhere (e.g., Greco \& Hayes, 2008). Yet practitioners taking this route should proceed with appropriate caution, recognizing that $\mathrm{MBI}$ is best considered an adjunct to-rather than a replacement for-other well-validated interventions at this level (Renshaw, 2020).

\section{Diversity and Developmental Considerations}

As described earlier, a growing body of intervention research supports the effectiveness of $\mathrm{MBI}$ with youth and their caregivers in both school and non-school settings. However, there is less evidence regarding the effectiveness of $\mathrm{MBI}$ for economically disadvantaged individuals and racial/ethnic minorities (DeLuca et al., 2018). This is especially true with youths and in schools (Black \& Fernando, 2014; Semple et al., 2010). One explanation from the National Health Interview Survey suggested that underrepresented populations were less likely to engage in MBI; however, the reasons are unclear (Olano et al., 2015). Similarly, DeLuca et al. (2018) indicated that the evidence for using MBI with this population is underdeveloped, which 
might limit the baseline frequency with which practitioners recommended it. That said, there is evidence to suggest that $\mathrm{MBI}$ are acceptable for underrepresented and marginalized populations, reporting higher program completion rates than other evidence-based treatments, along with high rates of continued use even after study termination (Dutton et al., 2013; Roth \& Robbins, 2004). MBI may also reduce risk for health disparities among underserved populations, by empowering individuals with effective social-emotional competencies that address these disparities (Woods-Giscombe \& Black, 2010). Given that school-based interventions are considered key for reducing disparities among undeserved youths (Fung et al., 2016), MBI integration in schools might be a pivotal strategy for reducing mental health care disparities for this population (Phan \& Renshaw, 2021).

To effectively adapt MBI with diverse populations in schools, it is crucial to make MBI culturally salient to optimize intervention effects (Chu \& Leino, 2017; Proulx et al., 2018). Elements of $\mathrm{MBI}$ should include addressing stress and its influence on health by incorporating techniques to encourage health promotion and self-care through the development of selfawareness and self-compassion (Woods-Giscombe \& Black, 2010). Incorporating these elements addresses psychological stress within its social context, which is especially important when supporting underrepresented populations' social-emotional needs. Moreover, practitioners implementing $\mathrm{MBI}$ in schools should consider that diverse youths may have different experiences with mind-body interventions depending on their gender identity, sexual identify, socioeconomic circumstances, and personal histories (Proulx et al., 2018). Becoming aware of how these identities and intersectionality may affect students and their caregivers is a touchstone for developing and implementing culturally-relevant MBIs in schools. 
Beyond general developmental considerations, appropriate use of $\mathrm{MBI}$ in schools may also require openness to cultural perspectives and adaptation for those with different cultural backgrounds or those presenting with specific disabling conditions. Since mindfulness originated in ancient Eastern Buddhist traditions, those with different cultural backgrounds might be cautious with mindfulness training and its perceived religious implications. WoodsGiscombé and Gaylord (2014) suggest adapting mindfulness training in ways that are viewed as compatible with the individual's values to handle such situations sensitively. For instance, practices such as benevolence and loving-kindness promoted through the practice of mindfulness are harmonious with principles that loving one's neighbor is the most important directive in the Christian tradition (Proulx et al., 2018). Those presenting with specific disabling conditions might also have difficulties with mindfulness training. Using techniques tailored to an individual's cognitive level may therefore be critical for facilitating the success of $\mathrm{MBI}$ in schools (Felver \& Singh, 2020). Given MBI exercises can use complex language, Neece et al. (2019) recommended that practitioners make their language less cognitively demanding. Finally, when considering mindfulness practices with special populations, we cannot overstate the importance of responding empathetically and transparently to their particular needs when developing adaptations to $\mathrm{MBI}$. Compassionate responding may serve an important function of rapport building, which is likely to encourage subsequent engagement with the intervention. Ultimately, diversity and developmental considerations should be actively attended to when implementing $\mathrm{MBI}$ in schools, not just passively acknowledged. 


\section{References}

Bender, S. L. (in press). Mindfulness-based parent training. Overview of mindfulness-based intervention research with parents and educators. In T. L. Renshaw \& S. R. Jimerson (Eds.), Using mindfulness to promote mental health in schools. Oxford.

Black, D. S. (2015). Mindfulness training for children and adolescents: A state-of-the-science review. In K. W. Brown, J. D. Creswell, \& R. M. Ryan (Eds.), Handbook of mindfulness: Theory, research, and practice (pp. 283-310). Guilford.

Black, D. S., \& Fernando, R. (2014). Mindfulness training and classroom behavior among lowerincome and ethnic minority elementary school children. Journal of Child and Family Studies, 23, 1242-1246. https://doi.org/10.1007/s10826-013-9784-4

Borquist-Conlon, D. S., Maynard, B. R., Brendel, K. E., \& Jarina, A.S. (2019). Mindfulness-based interventions for youth with anxiety: A systematic review and meta-analysis. Research on Social Work Practice, 29, 195-205. https://doi.org/10.1177/1049731516684961

Briere, J. (2011). Mindfulness inventory for children and adolescents. Unpublished measure. University of Southern California. https://mindfulscience.usc.edu/?usc research=psychometric-validation-of-the$\underline{\text { mindfulness-inventory-for-children-and-adolescents }}$

Broderick, P. (2013). Learning to BREATHE: A mindfulness curriculum for adolescents to cultivate emotion regulation, attention, and performance. New Harbinger.

Brown, K. W., \& Ryan, R. M. (2003). The benefits of being present: Mindfulness and its role in psychological well-being. Journal of Personality and Social Psychology, 84, 822-848. https://doi.org/10.1037/0022-3514.84.4.822 
Brown, K. W., West, A. M., Loverich, T. M., \& Biegel, G. M. (2011). Assessing adolescent mindfulness: Validation of an adapted Mindful Attention Awareness Scale in adolescent normative and psychiatric populations. Psychological Assessment, 23, 1023-1033.

\section{https://doi.org/10.1037/a0021338}

CREATE. (2020). Cultivating Awareness and Resilience in Education.

\section{https://createforeducation.org/care/}

Carsley, D., Khoury, B., \& Heath, N. L. (2018). Effectiveness of mindfulness interventions for mental health in schools: A comprehensive meta-analysis. Mindfulness, 9, 693-707. https://doi.org/10.1007/s12671-017-0839-2

Chu, J., \& Leino, A. (2017). Advancement in the maturing science of cultural adaptations of evidence-based interventions. Journal of Consulting and Clinical Psychology, 85, 4557. https://doi.org/10.1037/ccp0000145

Dariotis, J. K., Mirabal-Beltran, R., Cluxton-Keller, F., Gould, L. F., Greenberg, M. T., \& Mendelson, T. (2017). A qualitative exploration of implementation factors in a schoolbased mindfulness and yoga program: lessons learned from students and teachers. Psychology in the Schools, 54, 53-69. https://doi.org/10.1002/pits.21979

DeLuca, S. M., Kelman, A. R., \& Waelde, L. C. (2018). A systematic review of ethnoracial representation and cultural adaptation of mindfulness-and meditation-based interventions. Psychological Studies, 63, 117-129. https://doi.org/10.1007/s12646-018-

\section{$\underline{0452-z}$}

Dutton, M. A., Bermudez, D., Matas, A., Majid, H., \& Myers, N. L. (2013). Mindfulness-based stress reduction for low-income, predominantly African American women with PTSD 
and a history of intimate partner violence. Cognitive and Behavioral Practice, 20, 23-32.

\section{https://doi.org/10.1016/i.cbpra.2011.08.003}

Dunning, D. L., Griffiths, K., Kuyken, W., Crane, C., Foulkes, L., Parker, J., \& Dalgleish, T. (2019). The effects of mindfulness-based interventions on cognition and mental health in children and adolescents-A meta-analysis of randomized controlled trials. The Journal of Child Psychology and Psychiatry, 60, 244-258. https://doi.org/10.1111/jcpp.12980

de Bruin, E. I., Zijlstra, B. J., Geurtzen, N., van Zundert, R. M., van de Weijer-Bergsma, E., Hartman, E. E., Nieuwesteeg, A. M, Dunca, L. G., \& Bögels, S. M. (2014). Mindful parenting assessed further: Psychometric properties of the Dutch version of the Interpersonal Mindfulness in Parenting Scale (IM-P). Mindfulness, 5, 200-212.

\section{https://doi.org/10.1007/s12671-012-0168-4}

Duncan, L. G., Coatsworth, J. D., \& Greenberg, M. T. (2009). A model of mindful parenting: Implications for parent-child relationships and prevention research. Clinical Child and Family Psychology Review, 12, 255-270. https://doi.org/10.1007/s10567-009-0046-3

Droutman, V., Golub, I., Oganesyan, A., \& Read, S. (2018). Development and initial validation of the Adolescent and Adult Mindfulness Scale (AAMS). Personality and Individual Differences, 123, 34-43. https://doi.org/10.1016/i.paid.2017.10.037

Eklund, K., O’Malley, M., \& Meyer, L. (2017). Gauging mindfulness in children and youth: School-based applications. Psychology in the Schools, 54, 101-114. https://doi.org/10.1002/pits.21983

Felitti, V. J., Anda, R. F., Nordenberg, D., Williamson, D. F., Spitz, A. M., Edwards, V., \& Marks, J. S. (1998). Relationship of childhood abuse and household dysfunction to many of the 
leading causes of death in adults: The Adverse Childhood Experiences (ACE)

Study. American Journal of Preventive Medicine, 14, 245-258.

\section{https://doi.org/10.1016/S0749-3797(98)00017-8}

Felver, J. C., Doerner, E., Jones, J., Kaye, N. C., \& Merrell, K. W. (2013). Mindfulness in school psychology: Applications for intervention and professional practice. Psychology in the Schools, 50, 531-547. https://doi.org/10.1002/pits.21695

Felver, J. C., \& Singh, N. N. (2020). Mindfulness in the classroom: An evidence-based program to reduce disruptive behavior and increase academic engagement. New Harbinger.

Frantz, R., Hansen, S. G., \& Machalicek, W. (2018). Interventions to promote well-being in parents of children with autism: A systematic review. Review Journal of Autism and Developmental Disorders, 5, 58-77. https://doi.org/10.1007/s40489-017-0123-3

Fung, J., Kim, J. J., Jin, J., Chen, G., Bear, L., \& Lau, A. S. (2019). A randomized trial evaluating school-based mindfulness intervention for ethnic minority youth: Exploring mediators and moderators of intervention effects. Journal of Abnormal Child Psychology, 47, 1-19.

\section{https://doi.org/10.1007/s10802-018-0425-7}

Goodman, M. S., Madni, L. A., \& Semple, R. J. (2017). Measuring mindfulness in youth: Review of current assessments, challenges, and future directions. Mindfulness, 8, 1409-1420.

\section{https://doi.org/10.1007/s12671-017-0719-9}

Greco, L. A., \& Hayes, S. C. (Eds.). (2008). Acceptance \& mindfulness treatments for children and adolescents: A practitioner's guide. New Harbinger.

Greco, L. A., Baer, R. A., \& Smith, G. T. (2011). Assessing mindfulness in children and adolescents: Development and validation of the Child and Adolescent Mindfulness 
Measure (CAMM). Psychological Assessment, 23, 606-614.

\section{https://doi.org/10.1037/a0022819}

Greco, L. A., Lambert, W., \& Baer, R. A. (2008). Psychological inflexibility in childhood and adolescence: Development and evaluation of the Avoidance and Fusion Questionnaire for Youth. Psychological Assessment, 20, 93-102. https://doi.org/10.1037/1040-

\section{$\underline{3590.20 .2 .93}$}

Grossman, P. (2011). Defining mindfulness by how poorly I think I pay attention during everyday awareness and other intractable problems for psychology's (re)invention of mindfulness: Comment on Brown et al. (2011). Psychological Assessment, 23, 10341040. https://doi.org/10.1037/a0022713

Hayes, S. C., Strosahl, K. D., \& Wilson, K. G. (1999). Acceptance and commitment therapy: An experiential approach to behavior change. Guilford.

Johnson, C., Burke, C., Brinkman, S., \& Wade, T. (2017). Development and validation of a multifactor mindfulness scale in youth: The Comprehensive Inventory of Mindfulness Experiences-Adolescents (CHIME-A). Psychological Assessment, 29, 264-

\section{1. https://doi.org/10.1037/pas0000342}

Kabat-Zinn, J. (1994). Wherever you go, there you are: Mindfulness meditation in everyday life. Hyperion.

Kallapiran, K., Koo, S., Kirubakaran, R., \& Hancock, K. (2015). Effectiveness of mindfulness in improving mental health symptoms of children and adolescents: A meta-analysis. Child and Adolescent Mental Health, 20, 182-194. https://doi.org/10.1111/camh.12113

Klingbeil, D. A., \& Renshaw, T. L. (2018). Mindfulness-based interventions for teachers: A meta- 
analysis of the emerging evidence base. School Psychology Quarterly, 33, 501-511.

\section{https://doi.org/10.1037/spq0000291}

Klingbeil, D. A., Fischer, A. J., Renshaw, T. L., Bloomfield, B. S., Polakoff, B., Willenbrink, J. B., Copek, R. A., \& Chan, K. T. (2017). Effects of mindfulness-based interventions on disruptive behaviors: A meta-analysis of single-case research. Psychology in the Schools, 54, 70-87. https://doi.org/10.1002/pits.21982

Klingbeil, D. A., Renshaw, T. L., Willenbrink, J. B., Copek, R. A., Chan, K. T., Haddock, A., Yassine, J., \& Clifton, J. (2017). Mindfulness-based interventions with youth: A comprehensive meta-analysis of group design studies. Journal of School Psychology, 63, 77-103.

\section{https://doi.org/10.1016/j.jsp.2017.03.006}

Klingbeil, D. A., Fischer, A. J., \& Silberman, M. (in press). Overview of mindfulness-based intervention research with youth and students. In T. L. Renshaw \& S. R. Jimerson (Eds.), Mindfulness for improving mental health in schools. Oxford.

Kyriacou, C. (2001). Teacher stress: Directions for future research. Educational Review, 53, 2735. https://doi.org/10.1080/00131910120033628

Lawlor, M. S. (2016). Mindfulness and social emotional learning (SEL): A conceptual framework. In K. A. Schonert-Reichl \& R. W. Roeser (Eds.), Handbook of mindfulness in education (pp. 65-80). Springer.

Lawlor, M. S., Schonert-Reichl, K. A., Gadermann, A. M., \& Zumbo, B. D. (2014). A validation study of the Mindful Attention Awareness Scale adapted for children. Mindfulness, 5, 730-741. https://doi.org/10.1007/s12671-013-0228-4

Levinson, D. B., Stoll, E. L., Kindy, S. D., Merry, H. L., \& Davidson, R. J. (2014). A mind you can 
count on: Validating breath counting as a behavioral measure of mindfulness. Frontiers in Psychology, 5, Article 1202, 1-10. https://doi.org/10.3389/fpsyg.2014.01202

Linehan, M. M., Armstrong, H. E., Suarez, A., Allmon, D., \& Heard, H. L. (1991). Cognitivebehavioral treatment of chronically parasuicidal borderline patients. Archives of General Psychiatry, 48, 1060-1064. https://doi.org/10.1001/archpsyc.1991.01810360024003

Moore, R. C., Depp, C. A., Wetherell, J. L., \& Lenze, E. J. (2016). Ecological momentary assessment versus standard assessment instruments for measuring mindfulness, depressed mood, and anxiety among older adults. Journal of Psychiatric Research, 75, 116-123. https://doi.org/10.1016/j.jpsychires.2016.01.011

Neece, C. L., Chan, N., Klein, K., Roberts, L., \& Fenning, R. M. (2019). Mindfulness-based stress reduction for parents of children with developmental delays: understanding the experiences of Latino families. Mindfulness, 10, 1017-1030.

\section{https://doi.org/10.1007/s12671-018-1011-3}

Neff, K. D., Bluth, K., Tóth-Király, I., Davidson, O., Knox, M. C., Williamson, Z., \& Costigan, A. (2021). Development and validation of the Self-Compassion Scale for Youth. Journal of Personality Assessment, 103, 92-105. https://doi.org/10.1080/00223891.2020.1729774

Nock, M. K. (2007). Conceptual and design essentials for evaluating mechanisms of change. Alcoholism: Clinical and Experimental Research, 31, 4s-12s.

\section{https://doi.org/10.1111/i.1530-0277.2007.00488.x}

Olano, H. A., Kachan, D., Tannenbaum, S. L., Mehta, A., Annane, D., \& Lee, D. J. (2015). Engagement in mindfulness practices by US adults: Sociodemographic barriers. The Journal of Alternative and Complementary Medicine, 21, 100-102. 


\section{https://doi.org/10.1089/acm.2014.0269}

Phan, M. L., \& Renshaw, T. L. (2021). Guidelines for adapting mindfulness-based school interventions with underserved youth. OSF preprint. https://doi.org/10.31234/osf.io/rt65b

Proulx, J., Croff, R., Oken, B., Aldwin, C. M., Fleming, C., Bergen-Cico, D., Le T., \& Noorani, M. (2018). Considerations for research and development of culturally relevant mindfulness interventions in American minority communities. Mindfulness, 9, 361-370.

\section{https://doi.org/10.1007/s12671-017-0785-z}

Rayner, M., Hayes, L. L., \& Ciarrochi, J. (2017). Write your own DNA: A group program to help young people live with vitality and strength. https://thrivingadolescent.com

Renshaw, T. L. (2012). Mindfulness-based practices for crisis prevention and intervention. In S. E. Brock \& S. R. Jimerson (Eds.), Handbook of school crisis prevention and intervention (2nd ed., pp. 401-422). National Association of School Psychologists.

Renshaw, T. L. (2017). Preliminary development and validation of the Mindful Student Questionnaire. Assessment for Effective Intervention, 42, 168-175. https://doi.org/10.1177/1534508416678971

Renshaw, T. L., Fischer, A. J., \& Klingbeil, D. A. (2017). Mindfulness-based interventions in school psychology. Contemporary School Psychology, 21, 299-303.

\section{https://doi.org/10.1007/s40688-017-0166-6}

Roth, B., \& Robbins, D. (2004). Mindfulness-based stress reduction and health-related quality of life: Findings from a bilingual inner-city patient population. Psychosomatic Medicine, 66, 113-123. https://doi.org/10.1007/s40688-017-0166-6 
Semple, R. J., Lee, J., Rosa, D., \& Miller, L. F. (2010). A randomized trial of mindfulness-based cognitive therapy for children: Promoting mindful attention to enhance social-emotional resiliency in children. Journal of Child and Family Studies, 19, 218-229.

\section{https://doi.org/10.1007/s10826-009-9301-y}

Segal, Z. V., Williams, J. M. G., \& Teasdale, J. D. (2002). Mindfulness-based cognitive therapy for depression: A new approach to preventing relapse. Guilford.

Thayer, A. J., Sullivan, M., Larson, M., \& Cook, C. R. (in press). Overview of mindfulness-based intervention research with parents and educators. In T. L. Renshaw \& S. R. Jimerson (Eds.), Using mindfulness to promote mental health in schools. Oxford.

Thompson, B. L., \& Waltz, J. (2007). Everyday mindfulness and mindfulness meditation: Overlapping constructs or not? Personality and Individual Differences, 43, 1875-1885. https://doi.org/10.1016/j.paid.2007.06.017

Ullrich-French, S., Cox, A., Cole, A., Rhoades Cooper, B., \& Gotch, C. (2017). Initial validity evidence for the State Mindfulness Scale for Physical Activity with youth. Measurement in Physical Education and Exercise Science, 21, 177-189.

\section{https://doi.org/10.1080/1091367X.2017.1321543}

West, A. M., Sbraga, T. P., \& Poole, D. A. (2005). Measuring mindfulness in youth: Development of the Mindful Thinking and Action Scale for Adolescents. Unpublished manuscript. Central Michigan University.

West, A. M. (2008). Mindfulness and well-being in adolescence: An exploration of four mindfulness measures with an adolescent sample. Unpublished doctoral dissertation. Central Michigan University. 
Wong, K. F., Massar, S. A., Chee, M. W., \& Lim, J. (2018). Towards an objective measure of mindfulness: Replicating and extending the features of the breath-counting task. Mindfulness, 9, 1402-1410. https://doi.org/10.1007/s12671-017-0880-1

Woods-Giscombé, C. L., \& Black, A. R. (2010). Mind-body interventions to reduce risk for health disparities related to stress and strength among African American women: The potential of mindfulness-based stress reduction, loving-kindness, and the NTU therapeutic framework. Complementary Health Practice Review, 15, 115-131. https://doi.org/10.1177/1533210110386776

Woods-Giscombé, C. L., \& Gaylord, S. A. (2014). The cultural relevance of mindfulness meditation as a health intervention for African Americans: Implications for reducing stress-related health disparities. Journal of Holistic Nursing, 32, 147-160. https://doi.org/10.1177/0898010113519010

Zenner, C., Hermleben-Kurz, S., \& \& Walach, H. (2014). Mindfulness-based interventions in schools - a systematic review and meta-analysis. Frontiers in Psychology, 5, 1-20.

\section{https://doi.org/10.3389/fpsyg.2014.00603}

Zoogman, S., Goldberg, S. B., Hoyt, W. T., \& Miller, L. (2015). Mindfulness interventions with youth: A meta-analysis. Mindfulness, 6, 290-302. https://doi.org/10.1007/s12671-013$\underline{0260-4}$ 